\title{
Supplement to: Evaluating risk-prediction models using data from electronic health records
}

\author{
Le Wang, Pamela A. Shaw, Hansie M. Mathelier, \\ Stephen E. Kimmel, Benjamin French
}

This Supplement provides additional simulation results by summarizing the distribution of percent bias across simulated datasets. Recall that we calculated the percent bias in the estimates obtained using the misclassified outcomes to those obtained using the true outcomes. We present the results as density plots to show the entire distribution, along with a vertical bar from minus one to plus one standard deviation from the mean. Variability increased substantially as the misclassification rate among events $(p)$ increased, but decreased as the prevalence $(\pi)$ increased. Estimates based on the difference in the area under the receiver operating characteristic curve $(\Delta \mathrm{AUC})$ were generally more variable than those based on integrated discrimination improvement (IDI). 


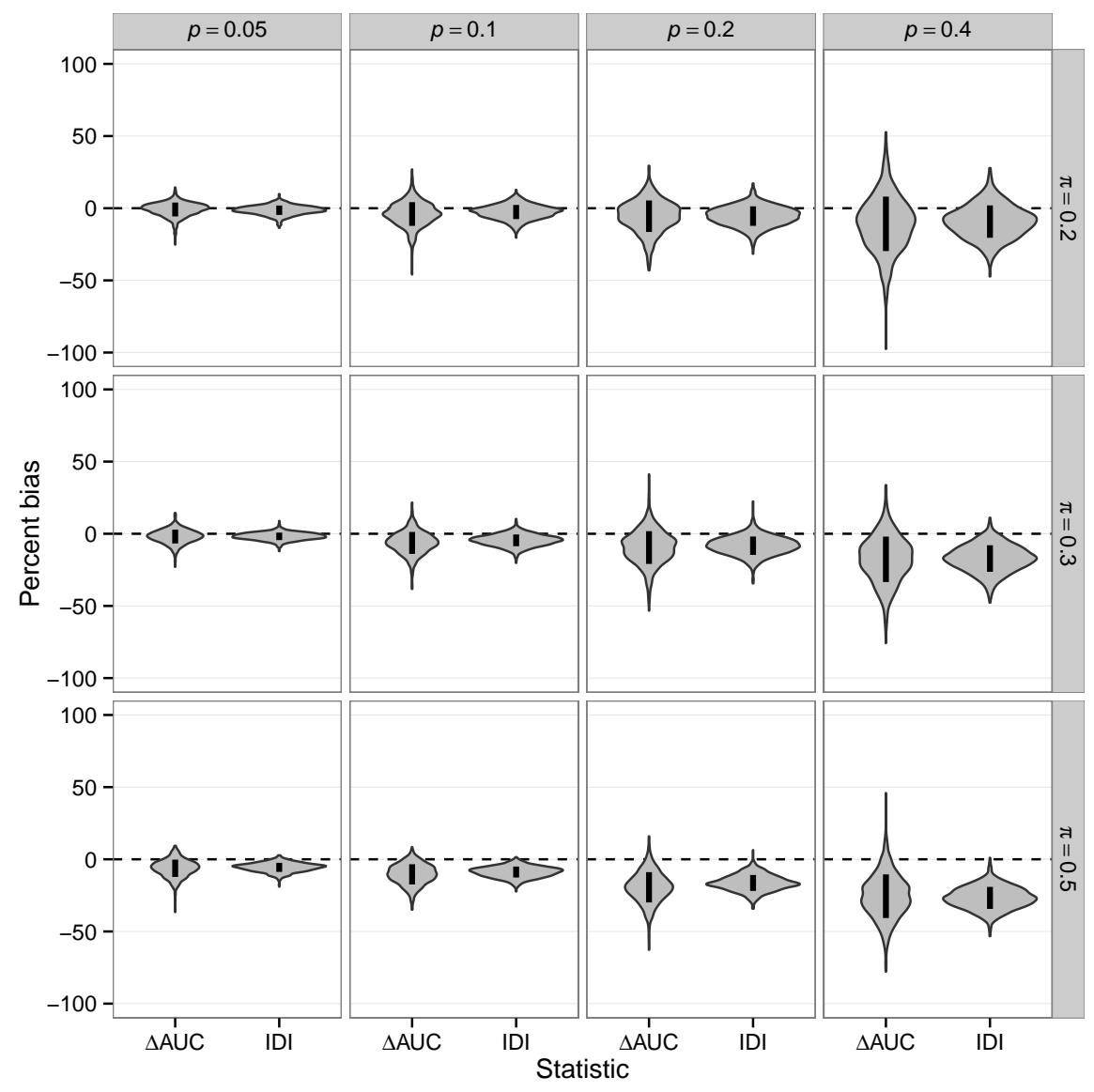

Supplementary Figure 1: Distribution of percent bias for the estimated $\Delta$ AUC and IDI under marker-independent outcome misclassification; $p$ denotes the misclassification rate among events; $\pi$ denotes the prevalence. 


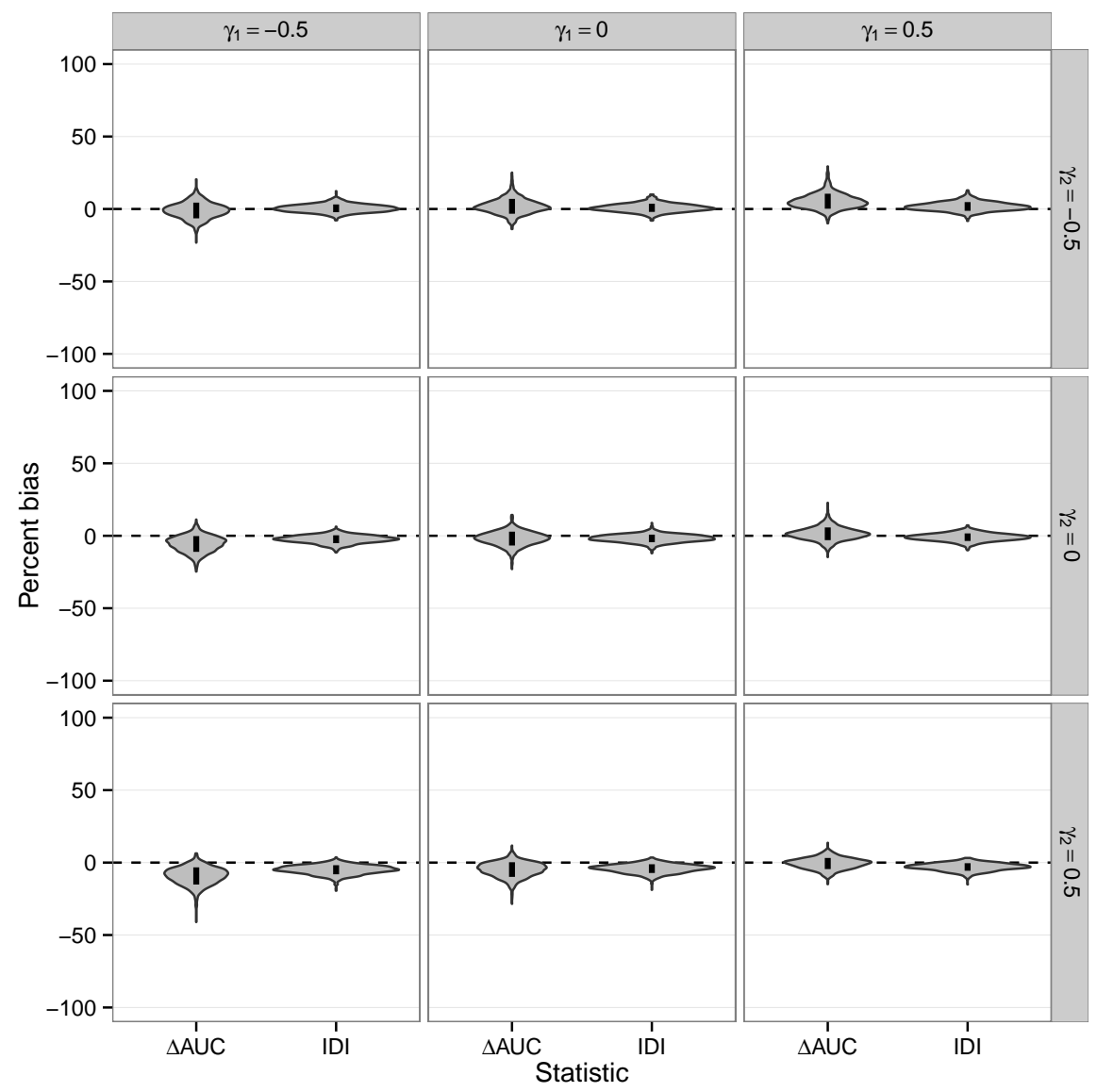

Supplementary Figure 2: Distribution of percent bias for the estimated $\Delta \mathrm{AUC}$ and IDI under marker-dependent outcome misclassification (prevalence $\pi=0.3$ and misclassification rate among events $p=0.05) ; \gamma_{1}$ and $\gamma_{2}$ correspond to the associations between markers $X$ and $Z$, respectively, and the log odds of misclassification among events. 


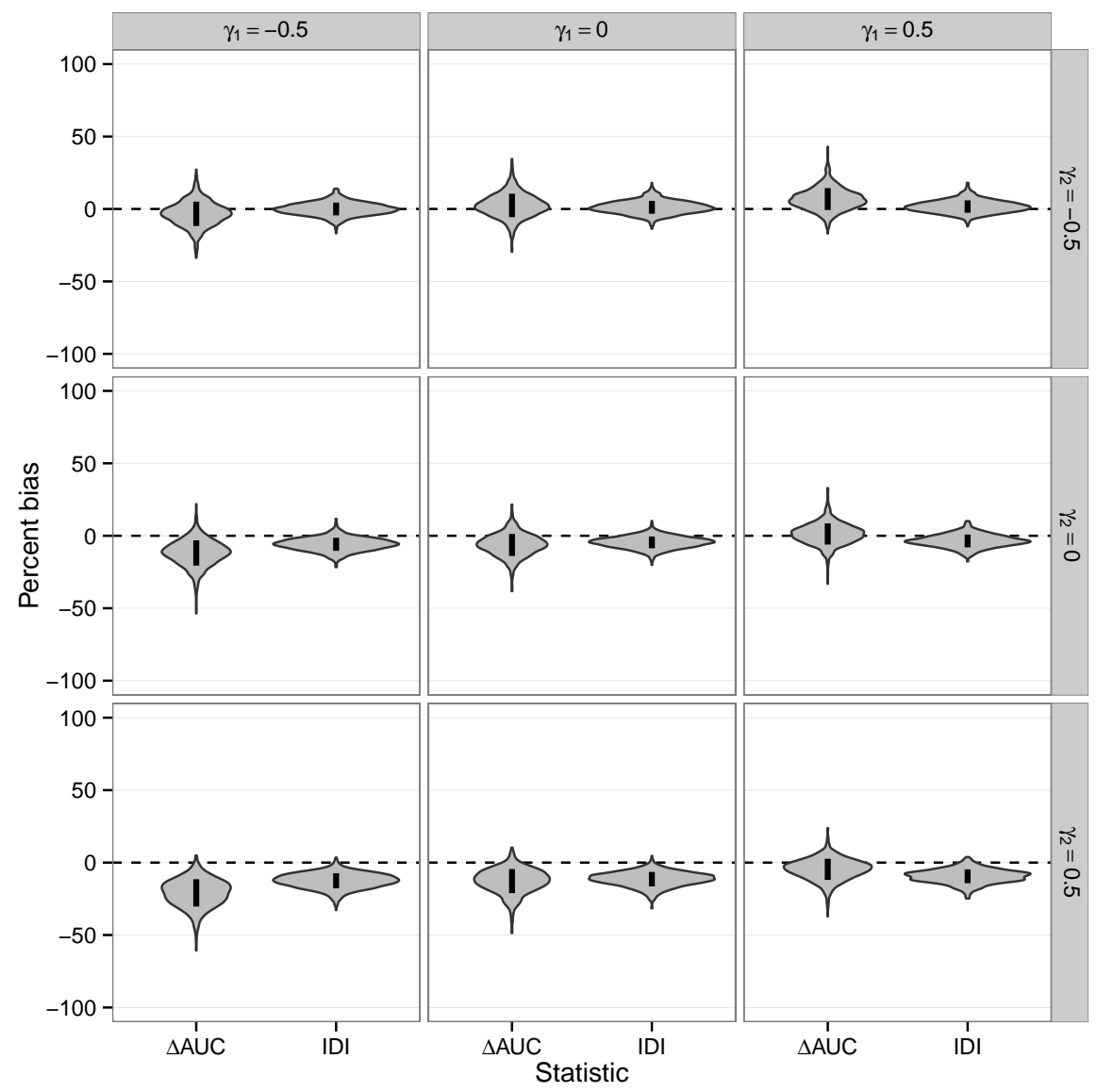

Supplementary Figure 3: Distribution of percent bias for the estimated $\Delta \mathrm{AUC}$ and IDI under marker-dependent outcome misclassification (prevalence $\pi=0.3$ and misclassification rate among events $p=0.1$ ); $\gamma_{1}$ and $\gamma_{2}$ correspond to the associations between markers $X$ and $Z$, respectively, and the log odds of misclassification among events. 


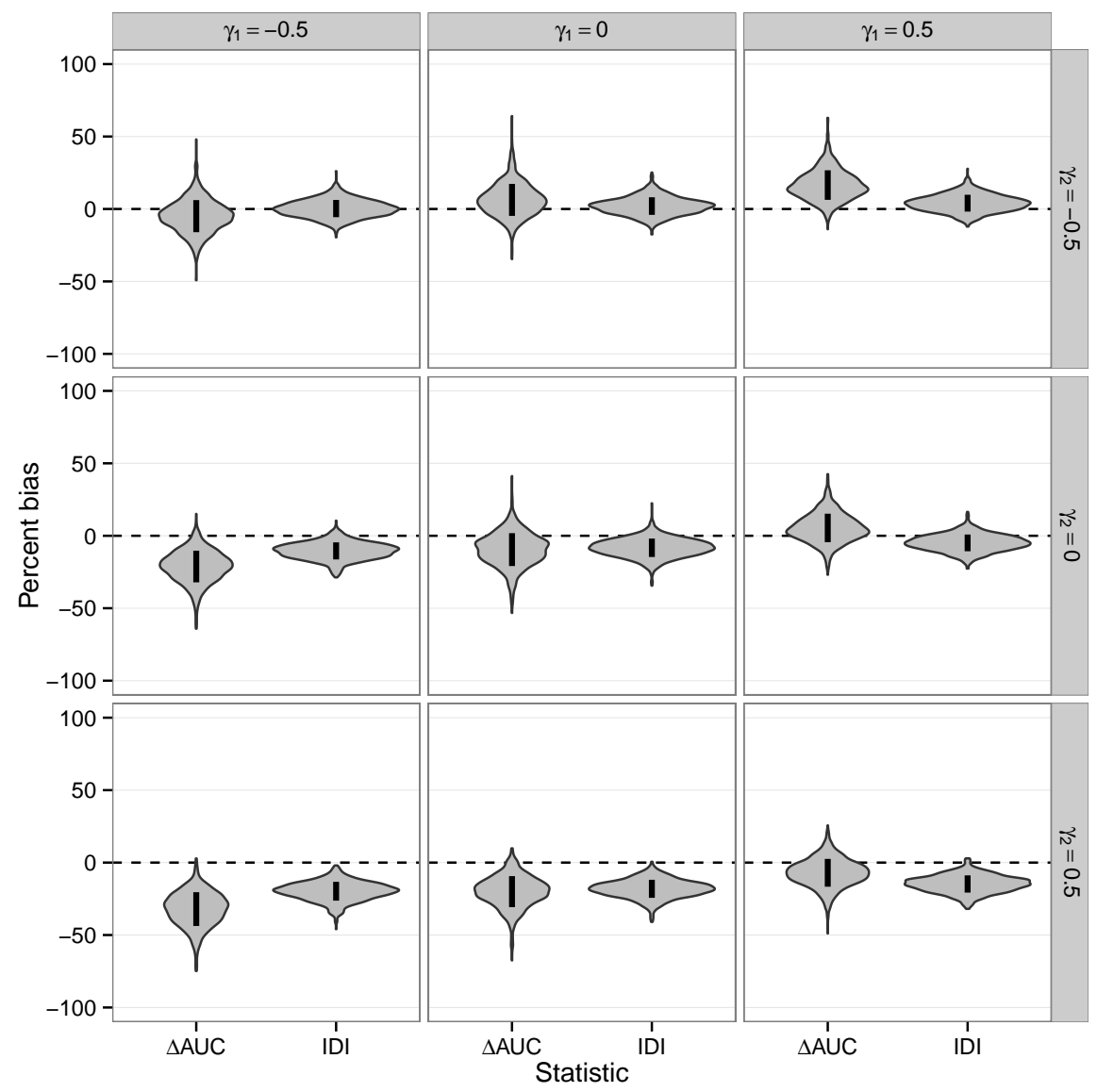

Supplementary Figure 4: Distribution of percent bias for the estimated $\Delta \mathrm{AUC}$ and IDI under marker-dependent outcome misclassification (prevalence $\pi=0.3$ and misclassification rate among events $p=0.2) ; \gamma_{1}$ and $\gamma_{2}$ correspond to the associations between markers $X$ and $Z$, respectively, and the log odds of misclassification among events. 


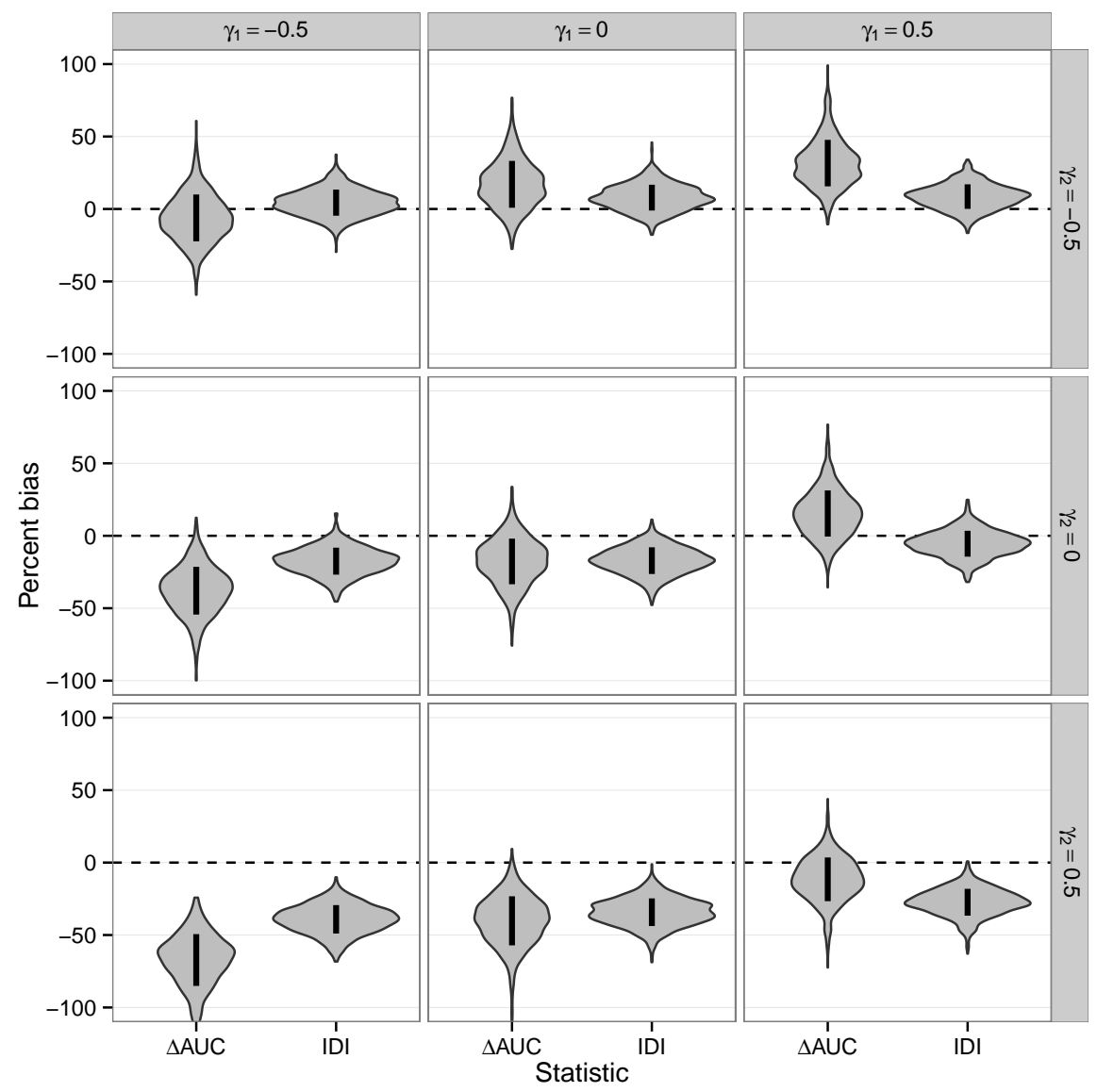

Supplementary Figure 5: Distribution of percent bias for the estimated $\Delta \mathrm{AUC}$ and IDI under marker-dependent outcome misclassification (prevalence $\pi=0.3$ and misclassification rate among events $p=0.4) ; \gamma_{1}$ and $\gamma_{2}$ correspond to the associations between markers $X$ and $Z$, respectively, and the log odds of misclassification among events. 